\section{Training your brain can improve food choice}

Regulation of craving (ROC) training - a mechanism-based intervention developed at Yale University, USA, by Hedy Kober and colleagues - improves food choices and reduces calorie intake, according to new research. In the present study, which included 1,528 participants, the authors found that individuals who had received ROC training were more likely than those who had not received the training to choose healthy foods over unhealthy foods

"In a prior meta-analysis we showed that craving for unhealthy foods is an important mechanism driving eating and weight gain," explains Kober, the corresponding author. "We also found that we can teach people to use cognitive strategies, which involve thinking about the negative consequences of unhealthy foods, to reduce their craving for unhealthy foods." With the aim of helping people to improve their eating and food choices, the team used the knowledge they gained during these prior studies to develop ROC training.

The ROC training was developed by combining methods from clinical psychology, experimental studies of emotion regulation and behavioural economics. Kober and colleagues tested the efficacy of ROC training on multiple dependent variables, including self-reported craving, willingness to pay for food (in \$US), food choices and calorie consumption. "This multi-modal approach allowed us to develop a more robust intervention - drawing insights from different fields - and to validate it on a variety of relevant outcomes," adds Kober.

The authors report that when faced with unhealthy food options, ROC training increased the participant's chance of selecting a healthy food choice by $5.4-11.2 \%$ and participants who received training indicated that they were willing to pay up to $\$ 1.50$ more for healthy foods. "The results of our study show that knowledge alone is not enough and that training in the implementation of knowledge is necessary to help people change their food choices," concludes Kober.

Alan Morris

ORIGINAL ARTICLE Boswell, R. G. et al. Training in cognitive strategies reduces eating and improves food choice. Proc. Natl Acad. Sci. USA https://doi.org/10.1073/pnas.1717092115 (2018)

\section{Highlights from SfE BES 2018}

This November, Glasgow, UK, hosted the Society for Endocrinology's annual meeting (SfE BES 2018), which saw endocrinologists from around the world gathering to discuss the latest advances in the field.

The first day opened with a fascinating symposium session on curing diabetes mellitus. Colin Dayan (Cardiff University, UK) outlined the need for immunotherapies for type 1 diabetes mellitus and gave an update on the trials that are currently underway. In addition, advances in clinical islet transplantation and the latest results on low-calorie diets for patients with type 2 diabetes mellitus were discussed.

In a symposium session entitled 'The most important 9 months; impact of maternal health', Susan Ozanne (University of Cambridge, UK) gave a detailed account of the effects of maternal diabetes mellitus on the child's health. 1 in 7 births are affected by gestational diabetes mellitus, and fetuses exposed to maternal diabetes mellitus in the womb seem to be at increased risk of cardiometabolic disease as a result of in utero epigenetic effects.

Ursula Kaiser (Brigham and Women's Hospital, USA) expertly delivered the first plenary of the conference. Kaiser discussed the neuroendocrine signals that trigger puberty and outlined how the timing of puberty onset can affect the risk of certain diseases (such as obesity and endometrial cancer) later in life.

Robin Peeters (Erasmus Medical Center, Netherlands) gave the Pitt-Rivers lecture, in which he discussed defining optimal thyroid function. Peeters outlined the challenges of defining thyroid functions in a range of situations, including during pregnancy; the fetus is dependent on maternal thyroxine, so maternal thyroid dysfunction can result in negative pregnancy outcomes, such as a preterm birth. On the final day of the conference, Maria-Christina Zennaro (INSERM, France) gave the European Medal Lecture on the molecular mechanisms of primary aldosteronism, which occurs in $11 \%$ of patients with hypertension.

SfE BES 2018 was a very enjoyable meeting, and I look forward to SfE BES 2019, which will be in Brighton, UK.

Claire Greenhill

FURTHER READING Agha-Jaffar, R. et al. Gestational diabetes mellitus: does an effective prevention strategy exist? Nat. Rev. Endocrinol. 12, 533-546 (2016) | Pozzilli, P. et al. Combination immunotherapies for type 1 diabetes mellitus. Nat. Rev. Endocrinol. 11, 289-297 (2015) | Korevaar, T. I. M. et al. Thyroid disease in pregnancy: new insights in diagnosis and clinical management. Nat. Rev. Endocrinol. 13, 610-622 (2017) 